\title{
Thresholds to arousing stimulation in the developing infant rhesus monkey'
}

\author{
GILBERT W. MEIER, J. F. KENNEDY CENTER, GEORGE PEABODY COLLEGE, NASHVILLE, TENNESSEE \\ RALPH J. BERGER, SPACE BIOLOGY LABORATORY, BRAIN RESEARCH INSTITUTE, LOS ANGELES. CALIF.
}

Thresholds of vocalization to electroshock were determined in infant and juvenile monkeys during random epochs of wakefulness, during high-voltage, slow-wave sleep, and during low-voltage, fast-wave sleep. These thresholds increased in all states over the first days of postnatal life and then decreased differentially for each of the states thereafter.

On the basis of psychophysiological indicants, supported by observations of overt behavior, only three states of vigilance have been identified in continuous recordings of infant and juvenile rhesus monkeys (Macaca mulatta): wakefulness (W); high-voltage, slowwave sleep (HVS); and low-voltage, fast-wave sleep (LVF). In the monkey (Meier \& Berger, 1965), as in the human (Roffwarg et al, 1966), the relative proportions and mean durations of these states change with the time since birth. One state, HVS, is functionally alike in infant and juvenile as attested to by similarities in reaction to selective state deprivation and in recovery following that deprivation, whereas LVF differs functionally as shown by age uniqueness on these same dimensions of selective state deprivation. In the infant, as contrasted with the juvenile, no sharp increase in frequency of onset of the selected state can be related to length of deprivation nor is the expected percentage of sleep time spent in LVF following the termination of deprivation altered. Further appraisal of functional similarities-dissimilarities in these vigilance states as related to age of $S$ was undertaken in the present experiment in which the thresholds to arousing stimulation during each of the three states were determined and related to developmental age.

Method

Three newly-born and three year-old juvenile rhesus monkeys were prepared for continuous polygraphic recording and for the aperiodic delivery of electroshock. Experimental manipulation lasted for 28 days for the infants and for at least two days for the juveniles. Presentation of continuously increasing, arousing stimulation was automatically programmed and was terminated by the monkey's vocalization or after a fixed duration at maximum intensity.

The maintenance and recording procedures were those used in the previous studies (Meier \& Berger, 1965; Berger \& Meier, 1966b). The vaginally-born infants $(\mathrm{N}=3)$ were separated from their mothers within $6 \mathrm{hr}$. of birth and reared thenceforth on mother surrogates upon which nursing bottles were mounted for ad lib feeding. The juvenile monkeys $(N=3), 12$ months of age, had been previously reared in the laboratory colony under standard conditions (Fleischman, 1963), but, during the course of this experimentation, were restrained in a primate chair (Muel Co.). For present purposes, all Ss were maintained in an electrostatically- and sound-shielded chamber (model 1201A, Industrial Acoustics Co.).

The continuous recording of electroencephalogram (EEG), electrooculogram (EOG), electromyogram (EMG), electrocardiogram (ECG), and impedance pneumogram (ZPG) was from hypodermic needle electrodes inserted subdermally and affixed with collodion. Vocalizations were picked up by a microphone placed about 12 in. from the monkey's head. The last were used to activate a voice-key and, thereby, the programming equipment.

The designation of the states of vigilance and the presentation of the electroshock at selected instances were accomplished by an automatic analyzer (Berger \& Meier, 1966a) which accepted information from the EMG and EOG for discrimination of the three states: W = high amplitude, irregular EMG with high frequency EOG (>1/15 sec.); HVS=EOG frequency at zero $(<$ $1 / 15$ sec.); and LVF = EMG amplitude at zero with high frequency EOG (> 1/15 sec.). The recordingstimulation sequence was as follows: The analyzer discriminated the state of vigilance during a 15-sec. episode. If the state continued for $20 \mathrm{sec}$., a tape programmer advanced and continued to do so as long as the state persisted. This programmer, in turn, activated the shock source (recording attenuator, model E7110A, Grason-Stadler, and shock generator, model E7110B, Grason-Stadler) at intervals 2-3 times that of the expected state-duration as reported in the normative studies. Once activated, the shock source increased its output continuously from $0.5 \mathrm{~mA}$ to $4.0 \mathrm{~mA}$ at which point the sequence was automatically terminated or until the $S$ vocalized, whichever occurred first. The maximum stimulus duration was 11 sec. The number of shock episodes was set by predetermined counters to 10 per $24 \mathrm{hr}$. interval for each of the three states of vigilance.

The recording-stimulation sequence began within $8 \mathrm{hr}$. of delivery for the three infant monkeys and after at least $48 \mathrm{hr}$. since preparation for the three juveniles. This 48-hr. period was one of adaptation during which each $S$ showed each state in characteristic pattern, frequency, and duration.

Although the recording and stimulation were automatically analyzed and programmed during the experi- 


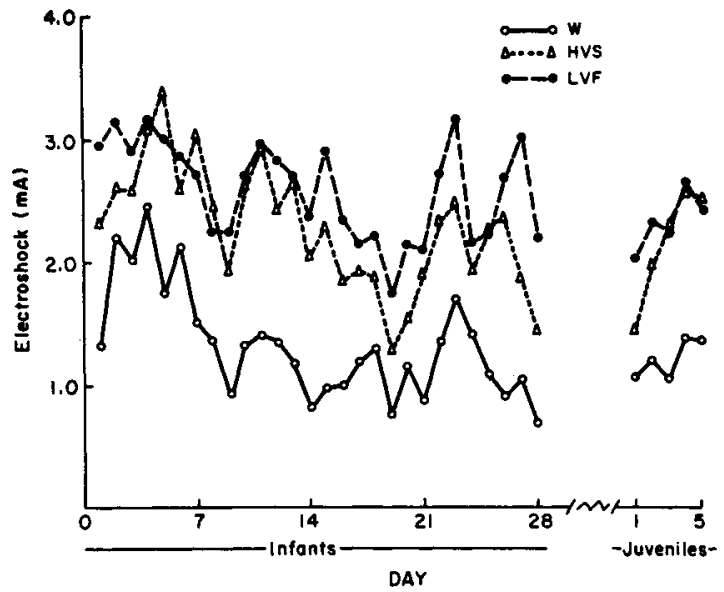

Fig. 1. Vocalization threshold in terms of $\mathrm{mA}$ of electroshock as related to developmental age and state of vigilance $(W \equiv$ wakefulness; HVS $\equiv$ sleep, high-voltage slow wave sleep; $L V F \equiv$ sleep, low-voltage fast wave sleep).

ment proper, the polygraphic recordings were later re-analyzed manually and the accuracy of the procedure verified. The ultimate comparisons, statistical and graphic, were made on these verified episodes and the associated shock levels as they appeared on the recording attenuator. In both comparisons, episodes in which the stimulation was automatically terminated were deleted. The effect of these episodes was that of adding a constant fraction to each of the mean values, especially those during the developmental periods of high thresholds. For the infants, this deletion meant that the analyses were made on five episodes for each state, for each day, for all animals.

\section{Results and Discussion}

The results are presented graphically in Fig. 1. The vocalization thresholds rose during the first four days after birth but then decreased until the fourth week in a manner unique for each state. The decline was most rapid for $W$ and least rapid for LVF. During the fourth and last week of recording on the infants, the shock levels increased during the two states of sleep, in particular. Overall, the thresholds between the three states collectively (LVF $>$ HVS $>W$ ), or between the two sleep states only (LVF>HVS) were statistically significant beyond the .001 level as determined by binomial tests. Likewise, the thresholds in the juveniles showed the same relation during the first two days of stimulation (LVF>HVS, $p<.001$ ) but the differences between the sleep states disappeared in the days thereafter as the threshold in HVS, at least, showed a progressive increase. In both groups, the vocalization was accompanied by polygraphically- defined wakefulness. In neither group was the transition from the selected sleep state to wakefulness necessarily, or even usually, through the other sleep state.

The relations between thresholds in the infant and juvenile monkeys are those reported for other nonhuman species (Siegel \& Langley, 1965) but are distinct from those reported for humans (Rechtschaffen et al, 1966). For the purposes of this investigation, however, the species differences are not so interesting as the similarity of the monkey to other non-human species and, more importantly, the overall similarity between the infantile and juvenile states. Only during the period when all thresholds were high, during the end of the first week of postnatal life, were HVS thresholds indistinguishable from those in LVF. This stage of high vocalization threshold in all states coincides with the transitional point described in the normative study when the percentage of LVF is at the maximum, the duration of individual periods of $W$ is at a minimum, and after which the individual durations of all three states of vigilance progressively increase to juvenile and adult levels. The rise in arousal thresholds in the infants during the fourth week and the juveniles after the second day suggests an habituation phenomenon. That the two age groups reached this point after experiences of differing duration may indicate that the rate of habituation is considerably less in the infant than in the adult; if not that the newborn shows little, if any, habituation (in the juvenile sense) but acquires this capacity after at least three weeks of postnatal development.

\section{References}

Berger, R. J., \& Meier, G. W. An automatic analyzer of states of vigilance. Psychophysiology, 1966a, 2, 141-145.

Berger, R. J., \& Meier, G. W. The effects of selective deprivation of states of sleep in the developing monkey. Psychophysiology, $1966 \mathrm{~b}, 2,354-371$.

Fleischman, R. W. The care of infant rhesus monkeys (Macaca mulatia). Lab. Anim. Care, 1963, 13, 703-710.

Meier, G. W., \& Berger, R. J. Development of sleep and wakefulness patterns in the infant rhesus monkey. Exp. Neurol., 1965 , $12,257-277$

Rechtschaffen, A., Hauri, P., \& Zeitlin, M. Auditory awakening thresholds in REM and NREM sleep stages. Percept. mot. Skills, 1966, 22, 927-942.

Roffwarg, H. P., Muzio, J. N., \& Dement, W. C. Ontogenetic development of the human sleep-dream cycle. Sciencc, 1966, 152, 604-619.

Siegel, J., \& Langley, T. D. Arousal threshold in the cat as a function of sleep phase and stimulus significance. Expcrientia, $1965,21,740$.

\section{Note}

1. The data reported here were collected in the Laboratory of Perinatal Physiology, National Institute of Neurological Diseases and Blindness, National Institutes of Health, United States Public Heal th Service, San Juan, Puerto Rico. 